\title{
Effectiveness of Gastric Cancer Screening on Gastric Cancer Incidence and Mortality in a Community-Based Prospective Cohort
}

\begin{tabular}{|c|}
\hline 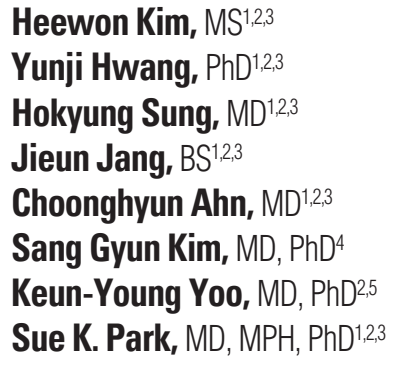 \\
\hline
\end{tabular}

Departments of ${ }^{1}$ Biomedical Science and ${ }^{2}$ Preventive Medicine, Seoul National University College of Medicine, Seoul, ${ }^{3}$ Cancer Research Center, Seoul National University, Seoul, ${ }^{4}$ Department of Internal Medicine and Liver Research Institute, Seoul National University College of Medicine, Seoul, ${ }^{5}$ The Armed Forces Capital Hospital, Seongnam, Korea

\author{
Correspondence: Sue K. Park, MD, MPH, PhD \\ Department of Preventive Medicine, \\ Seoul National University College of Medicine, \\ 103 Daehak-ro, Jongno-gu, Seoul 03080, Korea \\ Tel: 82-2-740-8338 \\ Fax: 82-2-747-4830 \\ E-mail: suepark@snu.ac.kr \\ Received January 25, 2017 \\ Accepted June 5, 2017 \\ Published Online June 9, 2017
}

\begin{abstract}
Purpose
This study was performed to investigate the effectiveness of gastric cancer (GC) screening methods in a community-based prospective cohort of the Korean Multi-center Cancer Cohort (KMCC) with over a 10-year follow-up.
\end{abstract}

\section{Materials and Methods}

A total 10,909 and 4,773 subjects from the KMCC with information on gastroendoscopy (GE) and upper gastrointestinal series (UGIS) were included in this study. Cox proportional hazard model adjusted for age, sex, Helicobacter pylori infection, cigarette smoking, and alcohol drinking was used to estimate the hazard ratios (HRs) and 95\% confidence interval (Cl).

\section{Results}

The GE screened subjects had almost half the risk of GC-specific death than that of unscreened subjects (HR, 0.58; $95 \% \mathrm{Cl}, 0.36$ to 0.94). Among the GC patients, GE screenees had a 2.24-fold higher survival rate than that of the non-screenees (95\% Cl, 1.61 to 3.11). In particular, GE screenees who underwent two or more screening episodes had a higher survival rate than that of the non-screenees (HR, 13.11; $95 \% \mathrm{Cl}, 7.38$ to 23.30). The effectiveness of GE screening on reduced GC mortality and increased survival rate of GC patients was better in elderly subjects ( $\geq 65$ years old) (HR, $0.47 ; 95 \% \mathrm{Cl}, 0.24$ to 0.95 and $\mathrm{HR}, 8.84 ; 95 \% \mathrm{Cl}, 3.63$ to 21.57 , respectively) than that in younger subjects ( $<65$ years old) (HR, 0.66; $95 \% \mathrm{Cl}, 0.34$ to 1.29 and $\mathrm{HR}, 1.83$; $95 \% \mathrm{Cl}, 1.24$ to 2.68 , respectively). In contrast, UGIS screening had no significant relation to GC mortality and survival.

\section{Conclusion}

The findings of this study suggest that a decreased GC-specific mortality and improved survival rate in GC patients can be achieved through GE screening.

\section{Introduction}

Gastric cancer (GC) is the fifth most common cancer, which is a major disease burden worldwide in recent years [1]. In Korea, GC is the second most common cancer after thyroid cancer, and GC has the third highest cancer death rate among all cancers [2]. The Korean government provides the National Cancer Screening Program (NCSP), which is a cancer screening program for five common cancers (i.e., stom-
Key words

Stomach neoplasms, Cohort studies, Mortality, Gastrointestinal endoscopy, Gastroscopy ach, liver, colorectal, breast, and cervix uteri) in the general population [3]. Under the NCSP, a GC screening program involving a biennial gastroendoscopy (GE) or upper gastrointestinal series (UGIS) screening was implemented in 2002 for people aged 40 years and older [4]. The lifetime GC screening rate under the NCSP was $83.4 \%$, and the GC screening rate based on the recommendation was $76.7 \%$ in 2014 [5]. In 2013, the participation rates in the NCSP for GC was $42.4 \%$ [5]. Among subjects who received GC screening, $72.6 \%$ and $28.4 \%$ underwent GE and UGIS screening, respec- 
tively, as a primary screening tool within the total Korean population [4].

Prior studies have evaluated the effects of GC screening among GC patients [6,7]. Those studies reported a higher likelihood of GC diagnoses in screenees than in non-screenees [6] and a relationship between the history of GC screening and advanced GC [7]. A case-control study also reported that GC patients admitted through a GE screening center had higher survival probability relative to those who were admitted through an outpatient clinic [8]. A retrospective cohort study using the NCSP data indicated that GE screening had higher sensitivity than that of UGIS screening for GC diagnosis [9]. However, that study was not focused on the associations between the GC screening methods and GC incidence, mortality, and survival. Moreover, studies on assessing the effectiveness of GC screening tools were rarely based on a population-based prospective cohort study.

Therefore, the aim of this study was to investigate the effectiveness of GC screening for assessing the GC incidence, GC-specific death, and GC survival probability in a community-based prospective cohort. Additionally, this study also evaluated the relative effectiveness of the two types of GC screening methods (GE and UGIS) used in Korea.

\section{Materials and Methods}

\section{Study design and study population}

The Korean Multi-center Cancer Cohort (KMCC) is a community-based prospective cohort with subjects from four areas of Korea (Haman, Chungju, Uljin, and Youngil). The KMCC data used in this study were collected from 1993 to 2004. Information on lifestyle factors and environmental factors were obtained from a questionnaire by well-trained interviewers. Anthropometric indices such as height, weight, waist circumference and hip circumference were also measured. The KMCC is explained in more detail in a previous report [10]. A total of 10,909 and 4,773 subjects had survey records on GE and / or UGIS screening information (screened and unscreened). The GE and UGIS screening status was classified into two groups (screened and unscreened) for each screening method. Each of the screened groups was further divided into three subgroups: low-frequency (one screening episode), high-frequency (two or more screening episodes) screening groups and unknown or missing group. There were 1,336 and 30 subjects with an unknown frequency for GE and UGIS screening, even though they had undergone screening.

The Institutional Review Boards of Seoul National Univer- sity Hospital (H-0110-084-002) and the National Cancer Center of Korea approved this KMCC-based study protocol (C-1310-082-528).

\section{Outcome and follow-up}

The main outcomes were GC incidence and GC-specific death. GC survival rate in post-diagnosis GC patients was an additional outcome variable. The incidences of GC (International Classification of Diseases for Oncology, third edition [ICD-O-3]: C16) were ascertained by linking the KMCC data to the National Cancer Registry until December 31, 2013, and GC-specific deaths (International Classification of Diseases, 10th edition [ICD10]: C16) were ascertained by linking the Nationwide Death Certificate databases until December 31, 2014.

\section{Assessment of exposure}

Information on screening was obtained from a selfreported questionnaire at baseline. Data on age (age at baseline), sex (men and women), cigarette smoking (never smoker, former smokers, and current smokers), and alcohol consumption (never drinker, former drinker, and current drinker) was obtained from the KMCC. Body mass index $\left(\mathrm{BMI}, \mathrm{kg} / \mathrm{m}^{2}\right)$ was calculated from the height and weight measured directly during the physical examination at base line. To determine Helicobacter pylori infection (positive and negative), immunoblot assay (Helico Blot 2.1, MP Biomedicals Asia Pacific, Singapore) was done with plasma samples collected at baseline. Our cohort only had collected blood and urine samples at baseline; thus, we could not do the Campylobacter-like organism test and urea breathe test which requires a biopsy and breathe sample. Nevertheless, this assay has several strengths. It detects the H. pylori IgG antibody which decreases slowly, and it indicates not only current infection but also past infection [11].

\section{Statistical analysis}

Comparison of screened and unscreened group characteristics was done by Student's $t$ test for continuous variables and chi-square test for categorical variables. We estimated hazard ratios (HRs) and 95\% confidence intervals (CI) with the Cox proportional hazard model after adjusting for age, sex, H. pylori infection (yes vs. no), cigarette smoking (yes vs. no), and alcohol consumption status (yes vs. no). $\mathrm{p}$ for trend were estimated by assigning scores for the categories of screening episodes from 1 for the lowest screening episode to 2 for the highest screening episode as a continuous variable. We conducted subgroup analysis with the frequencies of screening as the once group and the twice or more group. 
Table 1. Baseline characteristics of screenees and non-screenees by GE or UGIS screening

\begin{tabular}{|c|c|c|c|c|c|c|}
\hline \multirow[b]{2}{*}{ Characteristic } & \multicolumn{3}{|c|}{ GE screening } & \multicolumn{3}{|c|}{ UGIS screening } \\
\hline & $\begin{array}{l}\text { Unscreened } \\
(n=6,553)\end{array}$ & $\begin{array}{l}\text { Screened } \\
(n=4,356)\end{array}$ & p-value ${ }^{a)}$ & $\begin{array}{l}\text { Unscreened } \\
(n=2,758)\end{array}$ & $\begin{array}{l}\text { Screened } \\
(n=2,015)\end{array}$ & p-value ${ }^{a)}$ \\
\hline Proportion in total subjects $(\%)$ & 60.1 & 39.9 & & 57.8 & 42.2 & \\
\hline Age (yr) & $57.3 \pm 11.7$ & $58.0 \pm 10.3$ & $<0.001$ & $57.5 \pm 12.7$ & $58.9 \pm 10.2$ & $<0.001$ \\
\hline \multicolumn{7}{|l|}{ Sex } \\
\hline Male & $2,564(39.1)$ & $1,803(41.4)$ & 0.02 & $1,076(39.0)$ & $778(38.6)$ & 0.78 \\
\hline Female & $3,989(60.9)$ & $2,553(58.6)$ & & $1,682(61.0)$ & $1,237(61.4)$ & \\
\hline \multicolumn{7}{|l|}{ Body mass index $\left(\mathrm{kg} / \mathrm{m}^{2}\right)$} \\
\hline$<25$ & $3,856(58.9)$ & $2,377(54.6)$ & $<0.001$ & $1,486(53.9)$ & $1,002(49.7)$ & $<0.001$ \\
\hline$\geq 25$ & $1,973(30.1)$ & $1,237(28.4)$ & & $995(36.1)$ & $566(28.1)$ & \\
\hline Missing & $724(11.1)$ & $742(17.0)$ & & $277(10.0)$ & $447(22.2)$ & \\
\hline \multicolumn{7}{|l|}{ Education (schooling year) } \\
\hline$<12$ & $5,996(91.5)$ & $3,774(86.6)$ & $<0.001$ & $2,219(80.5)$ & $1,633(81.0)$ & 0.83 \\
\hline$\geq 12$ & $534(8.2)$ & $575(13.2)$ & & $532(19.3)$ & $376(18.7)$ & \\
\hline Missing & $23(0.4)$ & $7(0.2)$ & & $7(0.3)$ & $6(0.3)$ & \\
\hline \multicolumn{7}{|l|}{ Smoking status } \\
\hline Never & $4,168(63.6)$ & $2,693(61.8)$ & $<0.001$ & $1,791(64.9)$ & $1,277(63.4)$ & 0.22 \\
\hline Former & $621(9.5)$ & $634(14.6)$ & & 403 (14.6) & $334(16.6)$ & \\
\hline Current & $1,739(26.5)$ & $1,014(23.3)$ & & $554(20.1)$ & 393 (19.5) & \\
\hline Missing & $25(0.4)$ & $15(0.3)$ & & $10(0.4)$ & $11(0.6)$ & \\
\hline \multicolumn{7}{|l|}{ Alcohol drinking status } \\
\hline Never & $3,838(58.6)$ & $2,447(56.2)$ & 0.001 & $1,522(55.2)$ & $1,130(56.1)$ & 0.13 \\
\hline Former & $327(5.0)$ & $284(6.5)$ & & $194(7.0)$ & $108(5.4)$ & \\
\hline Current & $2,353(35.9)$ & $1,592(36.6)$ & & $1,014(36.8)$ & $754(37.4)$ & \\
\hline Missing & $35(0.5)$ & $33(0.8)$ & & $28(1.0)$ & $23(1.1)$ & \\
\hline
\end{tabular}

Values are presented as mean \pm standard deviation or number $(\%)$ unless otherwise indicated. GE, gastroendoscopy; UGIS, upper gastrointestinal series. ${ }^{a}$ Continuous variables were analyzed using the Student's $t$ test, categorical variables were analyzed using the chi-square test.

In addition, stratified analysis was also done by age $(<65$ and $\geq 65$ years old) and gastritis history (no and yes). To assess the combined or single effect by the two screening methods, we did a combination analysis between the groups stratified with various combinations according to the screening modalities (never screened, only screened by GE, only screened by UGIS, and screened by both GE and UGIS). p-values two sided and less than 0.05 were considered statistically significant. We performed all statistical analyses with SAS ver. 9.4 (SAS Institute, Cary, NC).

\section{Results}

Among the 10,909 subjects who had records on GE screening, there were 6,553 $(60.1 \%)$ who underwent GE screening and 4,356 (39.9\%) who did not undergo GE screening. Similarly, of the 4,773 subjects who had records on UGIS screening, there were 2,758 (57.8\%) who underwent UGIS screening and 2,015 (42.2\%) who did not undergo UGIS screening (Table 1).

Compared to the unscreened GE group, the GE screened group had more males and older subjects and also was more likely to have a higher BMI ( $\mathrm{p}<0.001)$ and higher education levels ( $p<0.001$ ) and had smokers (former and current smoking) $(\mathrm{p}<0.001)$ and alcohol drinkers (former and current drinking) $(\mathrm{p}=0.001)$. Within the UGIS group, the screenees consisted more of females and older subjects compared to the non-screenees $(\mathrm{p}<0.001)$ and were more likely to have a lower BMI $(\mathrm{p}<0.001)$. However, the other characteristics were not significantly different between the screened and unscreened UGIS groups (Table 1).

In the analysis for GE screening, 328 subjects were diagnosed with newly developed GC cases. Of those new cases in 130,666 person years, 107 patients died due to GC in 139,123 
Table 2. Risk of GC incidence, GC-specific death and overall survival

\begin{tabular}{|c|c|c|c|c|c|c|}
\hline & \multicolumn{3}{|c|}{ Gastroendoscopic screening } & \multicolumn{3}{|c|}{ Upper gastrointestinal tract series } \\
\hline & Person-years & $\begin{array}{l}\text { No. of } \\
\text { cases }\end{array}$ & $\begin{array}{c}\text { HR }^{\mathrm{a})} \\
(95 \% \mathrm{CI})\end{array}$ & Person-years & $\begin{array}{c}\text { No. of } \\
\text { cases }\end{array}$ & $\begin{array}{c}\text { HR }^{\mathrm{a})} \\
(95 \% \mathrm{CI})\end{array}$ \\
\hline \multicolumn{7}{|l|}{ General population } \\
\hline \multicolumn{7}{|l|}{ GC incidence } \\
\hline Never screened & 84,030 & 191 & 1.00 & 26,036 & 53 & 1.00 \\
\hline Ever screened & 46,636 & 137 & $1.21(0.94-1.54)$ & 18,222 & 50 & $0.83(0.52-1.33)$ \\
\hline \multicolumn{7}{|l|}{ Overall deaths } \\
\hline Never screened & 89,172 & 1,503 & 1.00 & 28,484 & 353 & 1.00 \\
\hline Ever screened & 50,626 & 682 & $0.87(0.79-0.95)$ & 20,266 & 198 & $0.84(0.70-1.00)$ \\
\hline \multicolumn{7}{|l|}{ GC-specific death } \\
\hline Never screened & 88,929 & 82 & 1.00 & 28,392 & 13 & 1.00 \\
\hline Ever screened & 50,194 & 25 & $0.58(0.36-0.94)$ & 20,001 & 9 & $0.91(0.36-2.33)$ \\
\hline \multicolumn{7}{|c|}{ Gastric cancer patients } \\
\hline \multicolumn{7}{|c|}{ Overall survivor rate } \\
\hline Never screened & 1,640 & 91 & 1.00 & 354 & 34 & 1.00 \\
\hline Ever screened & 793 & 87 & $2.24(1.61-3.11)$ & 217 & 32 & $1.36(0.76-2.45)$ \\
\hline
\end{tabular}

GC, gastric cancer; HR, hazard ratio; CI, confidence interval. a)Adjusted age, sex, Helicobacter pylori infection (positive vs. negative), cigarette smoking (yes vs. no), and alcohol consumption (yes vs. no).

Table 3. Risk of GC incidence, GC-specific death and overall survival by screening frequency

\begin{tabular}{|c|c|c|c|c|c|c|}
\hline & \multicolumn{3}{|c|}{ Gastroendoscopic screening } & \multicolumn{3}{|c|}{ Upper gastrointestinal tract series } \\
\hline & Person-years & $\begin{array}{l}\text { No. of } \\
\text { cases }\end{array}$ & $\begin{array}{c}\text { HR }^{\mathrm{a})} \\
(95 \% \mathrm{CI})\end{array}$ & Person-years & $\begin{array}{c}\text { No. of } \\
\text { cases }\end{array}$ & $\begin{array}{c}\text { HR }^{\mathrm{a})} \\
(95 \% \mathrm{CI})\end{array}$ \\
\hline \multicolumn{7}{|l|}{ General population } \\
\hline \multicolumn{7}{|l|}{ GC incidence } \\
\hline Never screened & 84,013 & 191 & 1.00 & 26,036 & 53 & 1.00 \\
\hline Screened once & 14,278 & 32 & $2.19(1.45-3.31)$ & 9,992 & 18 & $0.92(0.50-1.67)$ \\
\hline More than two times & 13,037 & 46 & $2.06(1.30-3.28)$ & 7,950 & 32 & $0.77(0.42-1.43)$ \\
\hline Unknown or missing b) & 19,320 & 59 & $0.83(0.60-1.15)$ & 281 & 0 & NA \\
\hline $\mathrm{p}$-trend & \multicolumn{3}{|r|}{$<0.001$} & & \multicolumn{2}{|r|}{0.35} \\
\hline \multicolumn{7}{|l|}{ GC-specific death } \\
\hline Never screened & 88,911 & 82 & 1.00 & 28,392 & 13 & 1.00 \\
\hline Screened once & 15,610 & 6 & $0.78(0.33-1.82)$ & 10,965 & 3 & $0.94(0.26-3.43)$ \\
\hline More than two times & 14,324 & 6 & $0.59(0.21-1.66)$ & 8,731 & 6 & $0.92(0.29-2.86)$ \\
\hline Unknown or missing ${ }^{\mathrm{b})}$ & 20,260 & 13 & $0.52(0.28-0.96)$ & 305 & 0 & NA \\
\hline $\mathrm{p}$-trend & \multicolumn{3}{|r|}{0.25} & & \multicolumn{2}{|r|}{0.83} \\
\hline \multicolumn{7}{|l|}{ Gastric cancer patients } \\
\hline \multicolumn{7}{|l|}{ Overall survivor rate } \\
\hline Never screened & 1,640 & 91 & 1.00 & 354 & 34 & 1.00 \\
\hline Screened once & 224 & 20 & $7.66(4.26-13.77)$ & 119 & 14 & $1.22(0.59-2.51)$ \\
\hline More than two times & 209 & 34 & $13.11(7.38-23.30)$ & 98 & 18 & $1.56(0.74-3.28)$ \\
\hline Unknown or missing ${ }^{\mathrm{b})}$ & 360 & 33 & $0.94(0.59-1.49)$ & 0 & 0 & NA \\
\hline $\mathrm{p}$-trend & \multicolumn{3}{|r|}{$<0.001$} & & \multicolumn{2}{|r|}{0.24} \\
\hline
\end{tabular}

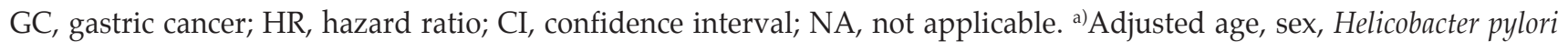
infection (positive vs. negative), cigarette smoking (yes vs. no), and alcohol consumption (yes vs. no), b)Unknown or missing frequency. 
Table 4. Risk of GC-specific death and overall survival by age

\begin{tabular}{|c|c|c|c|c|c|c|}
\hline & \multicolumn{3}{|c|}{ Age $<65$ yr } & \multicolumn{3}{|c|}{ Age $\geq 65$ yr } \\
\hline & Person-years & $\begin{array}{l}\text { No. of } \\
\text { cases }\end{array}$ & $\begin{array}{c}\mathrm{HR}^{\mathrm{a})} \\
(95 \% \mathrm{CI})\end{array}$ & Person-years & $\begin{array}{l}\text { No. of } \\
\text { cases }\end{array}$ & $\begin{array}{c}\mathrm{HR}^{\mathrm{a})} \\
(95 \% \mathrm{CI})\end{array}$ \\
\hline \multicolumn{7}{|l|}{ GC-specific death } \\
\hline Never screened & 67,595 & 36 & 1.00 & 21,334 & 46 & 1.00 \\
\hline Ever screened & 37,271 & 14 & $0.66(0.34-1.29)$ & 12,923 & 11 & $0.47(0.24-0.95)$ \\
\hline \multicolumn{7}{|c|}{ Survival rate in GC patients } \\
\hline Never screened & 1,112 & 73 & 1.00 & 528 & 18 & 1.00 \\
\hline Ever screened & 583 & 65 & $1.83(1.24-2.68)$ & 210 & 22 & $8.84(3.63-21.57)$ \\
\hline
\end{tabular}

GC, gastric cancer; HR, hazard ratio; CI, confidence interval. a)Adjusted age, sex, Helicobacter pylori infection (positive vs. negative), cigarette smoking (yes vs. no), and alcohol consumption (yes vs. no).

Table 5. Risk of GC-specific death and overall survival by history of gastritis

\begin{tabular}{|c|c|c|c|c|c|c|}
\hline & \multicolumn{3}{|c|}{ No gastritis } & \multicolumn{3}{|c|}{ Gastritis } \\
\hline & Person-years & $\begin{array}{l}\text { No. of } \\
\text { cases }\end{array}$ & $\begin{array}{c}\text { HR }^{\mathrm{a})} \\
(95 \% \mathrm{CI})\end{array}$ & Person-years & $\begin{array}{l}\text { No. of } \\
\text { cases }\end{array}$ & $\begin{array}{c}\mathrm{HR}^{\mathrm{a})} \\
(95 \% \mathrm{CI})\end{array}$ \\
\hline \multicolumn{7}{|l|}{ GC-specific death } \\
\hline Never screened & 79,782 & 73 & 1.00 & 6,624 & 8 & 1.00 \\
\hline Ever screened & 29,944 & 21 & $0.77(0.46-1.29)$ & 18,966 & 4 & $0.18(0.04-0.89)$ \\
\hline \multicolumn{7}{|c|}{ Survival rate in GC patients } \\
\hline Never screened & 1,471 & 81 & 1.00 & 111 & 7 & 1.00 \\
\hline Ever screened & 538 & 56 & $2.12(1.42-3.15)$ & 226 & 29 & $2.11(0.79-5.64)$ \\
\hline
\end{tabular}

GC, gastric cancer; HR, hazard ratio; CI, confidence interval. a)Adjusted age, sex, Helicobacter pylori infection (positive vs. negative), cigarette smoking (yes vs. no), and alcohol consumption (yes vs. no).

person years; 43 patients died due to causes other than GC, and 178 survived in 2,433 person years. In the UGIS screening analysis, there were 103 new GC cases diagnosed in 44,258 person years. Of those new cases, 22 patients died due to GC in 30,393 person years; 15 patients died due to other causes, and 66 survived in 571 person years.

The subjects in the GE screening group had a 0.87-fold reduced risk for overall death (95\% CI, 0.79 to 0.95$)$. Moreover, GE screenees had a much more reduced risk for GC-specific death (HR, 0.58 ; 95\% CI, 0.36 to 0.94$)$ relative to the GE nonscreenees. Among the patients diagnosed with GC, the GE screened subjects had a higher survival rate than that of the unscreened subjects (HR, 2.24; 95\% CI, 1.61 to 3.11). However, a significant difference was not found in the GC incidence between the screened and unscreened groups (Table 2).

GE screenees had a much more increased risk of GC incidence (screened once: HR, 2.19; 95\% CI, 1.45 to 3.31; screened more than two times: HR, 2.06; 95\% CI, 1.30 to 3.28; p-trend $<0.001$ ). The unknown or missing frequency of GE screening episodes prior to cohort enrollment was significantly associated with a lower risk of GC-specific death among the total participants (HR, $0.52 ; 95 \% \mathrm{CI}, 0.28$ to 0.96 ) and that was significantly associated with an improved survival rate among the GC patients (p-trend <0.001). Compared to non-screenees, subjects with one GE screening episode had a 7.66-fold higher survivor rate (95\% CI, 4.26 to 13.77), and those with two or more screening episodes had a much higher survivor rate (HR, 13.11; 95\% CI, 7.38 to 23.30) (Table 3).

The effectiveness of the GE screening in terms of the survival rate, which is the improvement of survival in the screenees compared to the non-screenees, was better in GC patients aged 65 years and older (HR, 8.84; 95\% CI, 3.63 to 21.57) than in GC patients aged less than 65 years (HR, 1.83; 95\% CI, 1.24 to 2.68) (Table 4). However, we could not find difference in the association between screening and GC survival according to gastritis history (Table 5).

In the analysis of the UGIS screening, there were no significant differences in the risk of GC incidence, GC-specific 
deaths, and survival rate between the screened and unscreened UGIS groups or between those screened once and those screened two or more times versus unscreened subjects (Tables 2 and 3). In addition, we did not observe any significant result from the combination analysis of the GC screening methods (S1 Table).

\section{Discussion}

The results suggest that GE screening was related to a decreased risk of GC-specific death based on the data from the community-based KMCC population over a minimum 10-year follow-up period. Compared to the unscreened group, the GE screening group had a higher survival rate in GC-diagnosed patients. Furthermore, the effectiveness of GE screening on improved survival in GC patients was more prominent in the older age group (age 65 years and older) than in the younger age group (aged less than 65 years).

To our knowledge, there is limited evidence based on randomized trial studies or large-scale cohort studies on the relationship of GC-specific death with GE or UGIE. Most previous studies were based on a case control study [12-15] or retrospective cohort study [16-18]. Among those studies, five Japanese studies $[12-14,16,18]$ have shown a lower risk of GC death associated with GC screening, which is a beneficial effect of the screening. However, two studies based on Western study populations did not show a significant association $[15,17]$. This inconsistency may be due to the lower GC incidences and GC-related mortality rates in the Venezuelan and U.S. study populations than those in the East Asian (Korea and Japan) study populations [1]. It may be caused by differences in the target disease in some of the studies (i.e., gastric cardia cancer vs. gastric cancer) [17] or by the absence of national guidelines or recommendations for GC screening in most countries other than Korea and Japan [19].

S2 Table shows a summary of the Japanese cohort studies which evaluated GC mortality in screened versus unscreened subjects by the modality $[18,20-23]$. Notably, the Japan Public Health Center-based prospective study (JPHC study) is comparable to our study [22]. Among the 42,150 subjects, the JPHC study results showed a $48 \%$ lower GC mortality among those who underwent photofluorography screening compared to the unscreened subjects [22]. It was similar to our results ( $51 \%$ reduction in GC mortality) despite of the use of different GC screening methods (i.e., gastric endoscopy and photofluorography, respectively).

Although our results did not show significant values in the analysis regarding UGIS, two Japanese studies suggested that UGIS also has a preventive effect for GC mortality
$[22,23]$. The difference in the observed effects of UGIS as GC screening in Japan and Korea may be due to differences in the GC screening guidelines and UGIS quality control (QC) programs of the two countries [24,25]. In Korea, the NCSP recommends biennial GE or UGIS screening as the first-line screening method for GC detection in people aged 40 years and older, while in Japan, annual photofluorography is recommended as the first-line screening method for GC detection in people in the same age group [24]. In addition, in 2007, Japan established municipal government programs to evaluate screening facilities and established a checklist for GC screening QC [26]. There have also been efforts to improve and standardize the quality of cancer screening in Korea. An evaluation of the quality of Korean and Japanese UGIS screening images revealed a higher QC score in Korea than in Japan (QC score, 73.31 in Korea; QC score, 58 in Japan) [27].

In the JPHC study [22], the overall GC incidence in the photofluorography screened population was not significantly different from that in the unscreened population (HR, 1.06; $95 \%$ CI, 0.90 to 1.25), which is consistent with our result. In our study, an increase in GE screening frequency was not associated with an increase in GC incidence; however, in another cohort study in Korea, repeated GE screening was associated with a lower GC incidence compared to less frequent screening [28]. That study included a highly selfmotivated population in which $99.6 \%(n=18,414)$ of the subjects voluntarily underwent GE screening, which may have led to its results being different from those in our study.

Several studies have examined the effectiveness of GC screening for increasing GC survival among GC patients $[7,8,29]$. However, based on study designs, only the results in one Japanese retrospective study, published in 2001, are directly comparable to the screened and unscreened groups in our study [29]. A significant beneficial effect associated with undergoing endoscopic examination within 2 years of GC diagnosis on GC survival was reported in the Japanese study $(\mathrm{p}<0.01)[29]$ and is consistent with the effect detected in our study. The higher GC survival probability in GC patients who underwent prior screening is presumably due to the ability of such screening to detect early-stage or localized GC. Our findings are consistent with prior studies performed in Japan and Korea [6,7,22].

Age stratified analyses were done in two previous studies in Japan $[13,22]$, and contradictory results were reported according to the screening modality. In a Japanese population-based case-control study [13], endoscopic screening in an older group led to a greater reduction in GC mortality reduction than that in a younger group (odds ratio [OR], 0.85; 95\% CI, 0.50 to 1.44 for patients aged 40-69 years; OR, 0.59 ; $95 \%$ CI, 0.37 to 0.95 for patients aged $70-79$ years). Our finding of an association between GE screening and a lower GC 
mortality is similar to the results of the Japanese study, even though the two age strata in our study (age less than 65 years and age 65 years and older) were different from those in the Japanese study. In contrast, a study reporting on the effectiveness of photofluorography-based UGIS screening showed that the younger group had a lower risk of death from GC compared to the older group (OR, $0.30 ; 95 \% \mathrm{CI}, 0.13$ to 0.72 for patients aged $40-49$ years; OR, 0.60 ; $95 \% \mathrm{CI}, 0.40$ to 0.88 for patients aged 50-59 years) [22]. Inconsistency among the results across these studies may be due to the use of different screening modalities (e.g., UGIS with photofluorography vs. endoscopy) and / or to differences in study populations (e.g., different age ranges, 40-79 years old vs. 50-69 years old).

According to previous studies, GE screening, but not UGIS screening, was beneficial for improving GC detection sensitivity [9] and diagnosis of localized and early-stage GC $[6,16,25]$. The results in these prior studies indirectly support our results, although the earlier results were not directly compared with our finding that GE had an effect on reduced death and increased survival; however, we did not observe any significant results in the UGIS analysis. Because of a relatively small sample size for UGIS and GC-specific death, the result must be interpreted cautiously.

In the combined analysis, we did not observe any significant association between the combined groups and GC-specific death. However, this result could be because only a few number of subjects had information on both screening methods.

This study has several limitations. First, this study may include a misclassification bias. Our study population includes individuals with differences between their GC diagnosis date in their medical records and the actual GC onset date. Second, symptomatic individuals were included in the study population. Third, we were unable to stratify our subjects by GC stage or histological subtype because our data source did not contain that information. Finally, we could not consider the duration of the screening interval for the screened groups. The survey questionnaire assessed two main factors: the experience of GC screening and its frequency. Because of the absence of the screening interval data, we only assessed the effect of the GE screening using frequency.

In conclusion, our community-based prospective cohort study in Korea, a country with a high GC incidence and mortality, suggests that GE screening has a significant effect on lowering GC-specific mortality. Moreover, GE screening improved the GC survival rate in GC patients, in particular, those aged 65 years and older.

\section{Electronic Supplementary Material}

Supplementary materials are available at Cancer Research and Treatment website (http:// www.e-crt.org).

\section{Conflicts of Interest}

Conflict of interest relevant to this article was not reported.

\section{Acknowledgments}

This work was supported by the National Research Foundation of Korea(NRF) grant funded by the Korea government(MSIP) (No. NRF-2016R1A2B4014552); the Education and Research Encouragement Fund of Seoul National University Hospital; and the Brain Korea 21 PLUS Program.

\section{References}

1. Ferlay J, Soerjomataram I, Dikshit R, Eser S, Mathers C, Rebelo $\mathrm{M}$, et al. Cancer incidence and mortality worldwide: sources, methods and major patterns in GLOBOCAN 2012. Int J Cancer. 2015;136:E359-86.

2. Jung KW, Won YJ, Oh CM, Kong HJ, Cho H, Lee DH, et al. Prediction of cancer incidence and mortality in Korea, 2015. Cancer Res Treat. 2015;47:142-8.

3. Yoo KY. Cancer control activities in the Republic of Korea. Jpn J Clin Oncol. 2008;38:327-33.

4. Lee S, Jun JK, Suh M, Park B, Noh DK, Jung KW, et al. Gastric cancer screening uptake trends in Korea: results for the National Cancer Screening Program from 2002 to 2011: a prospective cross-sectional study. Medicine (Baltimore). 2015;94:e533.
5. National Cancer Center. Cancer Facts \& Figures 2015 [Internet]. Sejong: Minister for Health and Welfare; 2015 [cited 2015 Jun 10]. Available from: https://www.ncc.re.kr/main.ncc? uri=english/sub04_FactsandFigures.

6. Choi KS, Jun JK, Suh M, Park B, Noh DK, Song SH, et al. Effect of endoscopy screening on stage at gastric cancer diagnosis: results of the National Cancer Screening Programme in Korea. Br J Cancer. 2015;112:608-12.

7. Lee H, Min BH, Lee JH, Son HJ, Kim JJ, Rhee JC, et al. Survival outcome associated with the screening interval for gastric cancer in Korea. Digestion. 2011;84:142-8.

8. Gong EJ, Ahn JY, Jung HY, Lim H, Choi KS, Lee JH, et al. Risk factors and clinical outcomes of gastric cancer identified by screening endoscopy: a case-control study. J Gastroenterol 
Hepatol. 2014;29:301-9.

9. Choi KS, Jun JK, Park EC, Park S, Jung KW, Han MA, et al. Performance of different gastric cancer screening methods in Korea: a population-based study. PLoS One. 2012;7:e50041.

10. Yoo KY, Shin HR, Chang SH, Lee KS, Park SK, Kang D, et al. Korean multi-center cancer cohort study including a biological materials bank (KMCC-I). Asian Pac J Cancer Prev. 2002;3: 85-92.

11. Lu CY, Kuo CH, Lo YC, Chuang HY, Yang YC, Wu IC, et al. The best method of detecting prior Helicobacter pylori infection. World J Gastroenterol. 2005;11:5672-6.

12. Matsumoto S, Yoshida Y. Efficacy of endoscopic screening in an isolated island: a case-control study. Indian J Gastroenterol. 2014;33:46-9.

13. Hamashima C, Ogoshi K, Okamoto M, Shabana M, Kishimoto T, Fukao A. A community-based, case-control study evaluating mortality reduction from gastric cancer by endoscopic screening in Japan. PLoS One. 2013;8:e79088.

14. Fukao A, Tsubono Y, Tsuji I, HIsamichi S, Sugahara N, Takano A. The evaluation of screening for gastric cancer in Miyagi Prefecture, Japan: a population-based case-control study. Int J Cancer. 1995;60:45-8.

15. Pisani P, Oliver WE, Parkin DM, Alvarez N, Vivas J. Case-control study of gastric cancer screening in Venezuela. Br J Cancer. 1994;69:1102-5.

16. Matsumoto S, Ishikawa S, Yoshida Y. Reduction of gastric cancer mortality by endoscopic and radiographic screening in an isolated island: a retrospective cohort study. Aust J Rural Health. 2013;21:319-24.

17. Cooper GS, Yuan Z, Chak A, Rimm AA. Association of prediagnosis endoscopy with stage and survival in adenocarcinoma of the esophagus and gastric cardia. Cancer. 2002;95:32-8.

18. Hosokawa O, Miyanaga T, Kaizaki Y, Hattori M, Dohden K, Ohta K, et al. Decreased death from gastric cancer by endoscopic screening: association with a population-based cancer registry. Scand J Gastroenterol. 2008;43:1112-5.

19. Dan YY, So JB, Yeoh KG. Endoscopic screening for gastric cancer. Clin Gastroenterol Hepatol. 2006;4:709-16.

20. Inaba S, Hirayama H, Nagata C, Kurisu Y, Takatsuka N,
Kawakami N, et al. Evaluation of a screening program on reduction of gastric cancer mortality in Japan: preliminary results from a cohort study. Prev Med. 1999;29:102-6.

21. Kaneko S, Tamakoshi A, Ohno Y, Mizoue T, Yoshimura T; JACC Study Group. Menstrual and reproductive factors and the mortality risk of gastric cancer in Japanese menopausal females. Cancer Causes Control. 2003;14:53-9.

22. Lee KJ, Inoue M, Otani T, Iwasaki M, Sasazuki S, Tsugane S, et al. Gastric cancer screening and subsequent risk of gastric cancer: a large-scale population-based cohort study, with a 13-year follow-up in Japan. Int J Cancer. 2006;118:2315-21.

23. Miyamoto A, Kuriyama S, Nishino Y, Tsubono Y, Nakaya N, Ohmori $\mathrm{K}$, et al. Lower risk of death from gastric cancer among participants of gastric cancer screening in Japan: a population-based cohort study. Prev Med. 2007;44:12-9.

24. Hamashima C, Shibuya D, Yamazaki H, Inoue K, Fukao A, Saito $\mathrm{H}$, et al. The Japanese guidelines for gastric cancer screening. Jpn J Clin Oncol. 2008;38:259-67.

25. Tashiro A, Sano M, Kinameri K, Fujita K, Takeuchi Y. Comparing mass screening techniques for gastric cancer in Japan. World J Gastroenterol. 2006;12:4873-4.

26. Higashi T, Machii R, Aoki A, Hamashima C, Saito H. Evaluation and revision of checklists for screening facilities and municipal governmental programs for gastric cancer and colorectal cancer screening in Japan. Jpn J Clin Oncol. 2010;40: 1021-30.

27. Oh HK, Kim JM, Kim CG, Park YS, Seon JR, Choi IS. Quality control of upper gastrointestinal seriese (UGIS) by the Image Quality Evaluation Table of Korea and Japan. J Radiol Sci Technol. 2011;34:277-85.

28. Nam SY, Choi IJ, Park KW, Kim CG, Lee JY, Kook MC, et al. Effect of repeated endoscopic screening on the incidence and treatment of gastric cancer in health screenees. Eur J Gastroenterol Hepatol. 2009;21:855-60.

29. Mori Y, Arita T, Shimoda K, Yasuda K, Yoshida T, Kitano S. Effect of periodic endoscopy for gastric cancer on early detection and improvement of survival. Gastric Cancer. 2001;4: 132-6. 